\title{
Management of Cavernous Sinus Chondroma in the Pediatric Population: Case Report and Comprehensive Literature Review
}

\author{
Carlos Roberto Massella, MD, Yasmeen Elsawaf, BS², Sabino Luzzi, MD ${ }^{3,4}$, Paulo Mácio Porto de Melo, \\ $M D^{1}$ and Samer K Elbabaa, MD, FAANS, FAAP, FACS ${ }^{2 *}$
}

${ }^{1}$ Department of Neurosurgery, Sao Paulo Military Area Hospital, Brazilian Army, Sao Paulo, Brazil

${ }^{2}$ Department of Pediatric Neurosurgery, Arnold Palmer Hospital for Children, Orlando, USA

${ }^{3}$ Department of Clinical-Surgical, Diagnostic Pediatric Sciences, Neurosurgery Unit, University of Pavia, Pavia, Italy

${ }^{4}$ Department of Surgical Sciences, Neurosurgery Unit, Fondazione IRCCS Policlinico San Matteo, Pavia, Italy

*Corresponding author: Samer K Elbabaa, MD, FAANS, FAAP, FACS, Department of Pediatric Neurosurgery, Arnold Palmer Hospital for Children, 92 W Miller St, Orlando, FL 32806, USA, Tel: 321-841-3050

\begin{abstract}
Introduction: Resection of a cavernous sinus tumor may be performed utilizing three primary approaches: The middle cranial fossa epidural approach, the combined transsylvian-subtemporal approach, and the medial transsphenoidal approach.

Case: Herein, we describe an exceedingly rare cavernous sinus chondroma in an 18-year-old male who presented with a history of binocular diplopia and trochlear nerve palsy. Brain CT scan detected a left sided cavernous sinus calcified mass and the MRI confirmed an enhancing lesion in the left cavernous sinus and the patient subsequently underwent successful resection of the mass via a pre-temporal trans-cavernous approach. Histopathological biopsy provided the definitive diagnosis of a cavernous sinus chondroma.

Conclusion: In this report, we discuss diagnostic imaging and review the current literature on management strategies for the treatment of cavernous sinus chondromas in the pediatric population.
\end{abstract}

\section{Keywords}

Cavernous sinus, Chondroma, Middle cranial fossa, Transsylvian-subtemporal approach, Sellar chondroma, Pretemporal transcavernous resection

\section{Introduction}

Intracranial chondromas are rare benign tumors with a prevalence of only $0.2-0.3 \%$ in the general population [1]. Such tumors are most often diagnosed in the third decade of life and have no gender predilection [2]. Chondromas predominantly arise at the base of the skull in the sellar region from embryonic chondrocytic cell remnants, and are also common in the falx and convexity dura [3-5]. Though rare, chondromas may present in the cavernous sinus as well.

The cavernous sinus (CS) is a dural venous plexus traversed by neurovascular structures including the cavernous segment of the internal carotid artery (ICA), oculomotor nerve, trochlear nerve, abducent nerve and ophthalmic and maxillary divisions of the trigeminal nerve. Clinical presentation of CS lesions may vary in accordance with neurovascular disturbances; CS syndrome is characterized by paresis of one or more cranial nerves (III-VI), with or without associated ophthalmoplegia $[6,7]$. Common tumors of the cavernous sinus include meningiomas, neurogenic tumors, hemangiomas, epidermoid cysts, dermoid cysts, schwannomas, and pituitary adenomas [6,8].

Germ cell tumors and tuberculomas are also rare entities found in the cavernous sinus in the pediatric population [9-11]. Although chondromas of the cavernous sinus are extremely rare in the pediatric population; common lesions in the cavernous sinus include B cell lymphoma, Hodgkin lymphoma, Burkitt lymphomas as well as Tolosa-Hunt syndrome (Table 1) [8,12]. A study by $\mathrm{Su}$ J, et al., reported one chondroma in a case series

Citation: Massella CR, Elsawaf Y, Luzzi S, Melo PMP, Elbabaa SK (2020) Management of Cavernous Sinus Chondroma in the Pediatric Population: Case Report and Comprehensive Literature Review. Neurosurg Cases Rev 3:048. doi.org/10.23937/2643-4474/1710048

Accepted: December 04, 2020; Published: December 06, 2020

Copyright: (C) 2020 Massella CR, et al. This is an open-access article distributed under the terms of the Creative Commons Attribution License, which permits unrestricted use, distribution, and reproduction in any medium, provided the original author and source are credited. 
Table 1: Historical cavernous sinus lesions.

\begin{tabular}{|l|l|l|l|l|}
\hline Author & Year & Age & Sex & Diagnosis \\
\hline Ceyhan [24] & 1994 & 4 years & Female & B-cell lymphoma \\
\hline Kalina [25] & 1996 & 4 years & Male & Burkitt lymphoma \\
\hline Ganesan [26] & 1996 & 13 years & Male & Tolosa-Hunt syndrome \\
\hline Del Toro [27] & 2001 & 10 years & Male & Tolosa-Hunt syndrome \\
\hline Yeung [28] & 2004 & 9 years & Male & Tolosa-Hunt syndrome \\
\hline Lee [29] & 2004 & 9 years & Male & Burkitt lymphoma \\
\hline Seixas [30] & 2006 & 11 years & Male & Burkitt lymphoma \\
\hline Zanus [31] & 2009 & 8 years & Female & Tolosa-Hunt syndrome \\
\hline Alioglu [32] & 2009 & 11 years & Male & Hodgkin lymphoma \\
\hline Akinci [33] & 2009 & 10 years & Male & B-cell lymphoma \\
\hline Demirkaya [34] & 2010 & 4 years & Male & B-cell lymphoma \\
\hline Cerisola [35] & 2011 & 11 years & Male & Tolosa-Hunt syndrome \\
\hline Kang [36] & 2011 & 7 years & Male & Tolosa-Hunt syndrome \\
\hline Litkowski [37] & 2011 & 11 years & Male & Hemangioma \\
\hline Benzohra [38] & 2011 & 4 years & Female & $\begin{array}{l}\text { THS intracavernous carotid artery compression, With consecutive } \\
\text { CV accident and hemiplegia }\end{array}$ \\
\hline & & & & \\
\hline
\end{tabular}

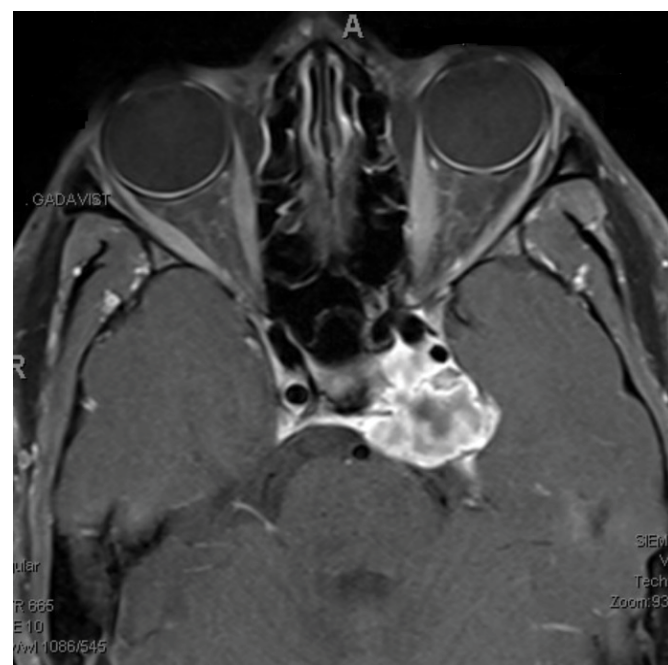

A

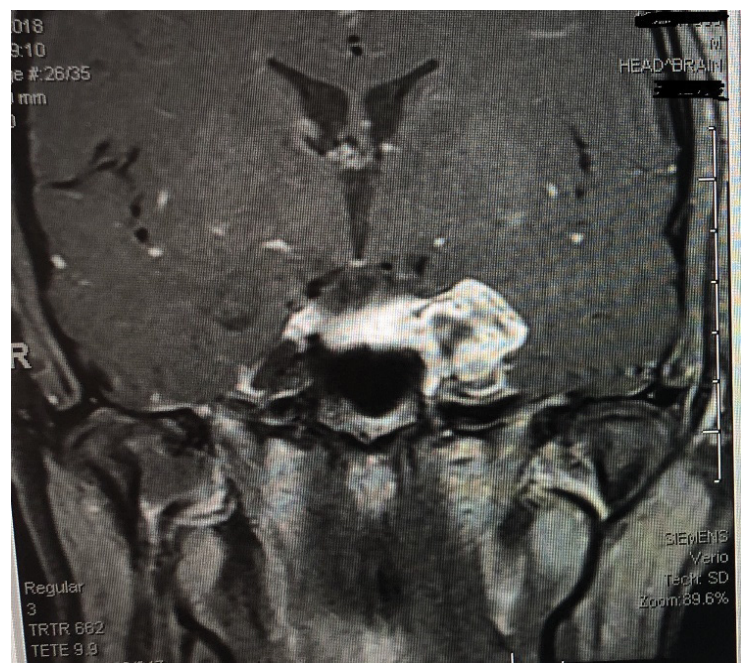

B

Figure 1: $(A, B)$ Pre-operative axial and coronal brain MRI imaging using T1 sequence with contrast shows the heavily enhancing mass arising from the left cavernous sinus with mass effect on mesial temporal lobe and displacement of the ICA artery.

of 31 cavernous sinus tumors in adults [12]. Another case report detailed the management of a chondroma in a 40 -year-old patient $[8,13]$. Further management of intracavernous tumors is described over an 11-year time period by Sepehrnia, et al., with mention of only one Chondroma [14].

In the case described below, we highlight a pediatric cavernous sinus chondroma and describe the incidence and management of such tumors.

\section{Case Description}

\section{History and examination}

An 18-year-old male with no significant past medical history presented with a three week history of worsen- ing left monocular diplopia and compensatory head tilt. Five months prior to presentation left sided neglect was noted by his father. He denied any associated symptoms including headaches, nausea, fatigue, bone/back/ neck pain, or changes in cognition/speech/hearing. Initial neurological examination revealed left abducens nerve palsy and partial left trochlear nerve palsy, as well as anisocoria with left pupil larger than the right. The patient underwent brain and orbital magnetic resonance imaging (MRI) revealing a $27.3 \times 29.0 \times 18.6 \mathrm{~mm}$ left cavernous sinus mass arising from the petrous apex with extension into the cavernous sinus and the Dorello's canal; such findings are consistent with the presenting symptoms (Figure $1 \mathrm{~A}$ and Figure $1 \mathrm{~B}$ ). The CT scan confirmed a calcified mass (Figure 2A and Figure 2B). 


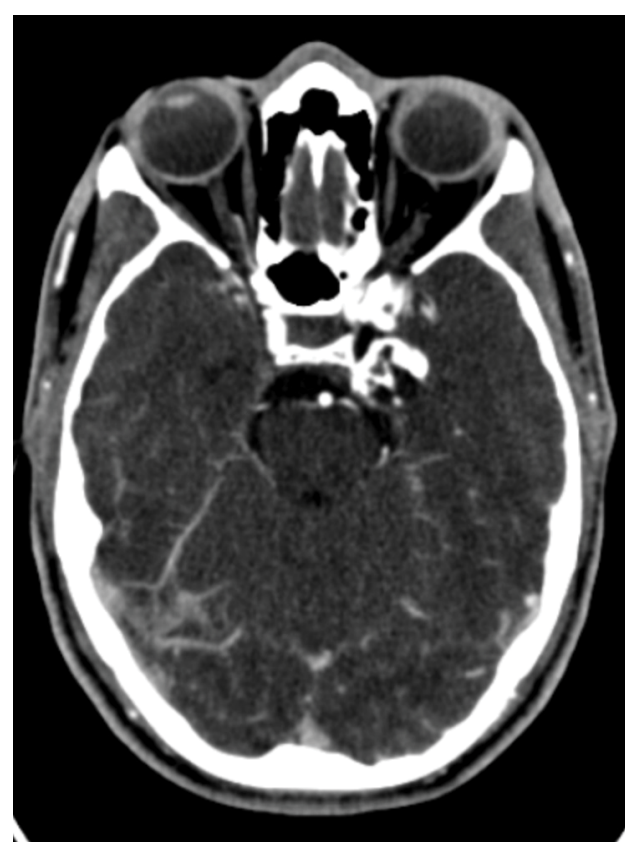

A

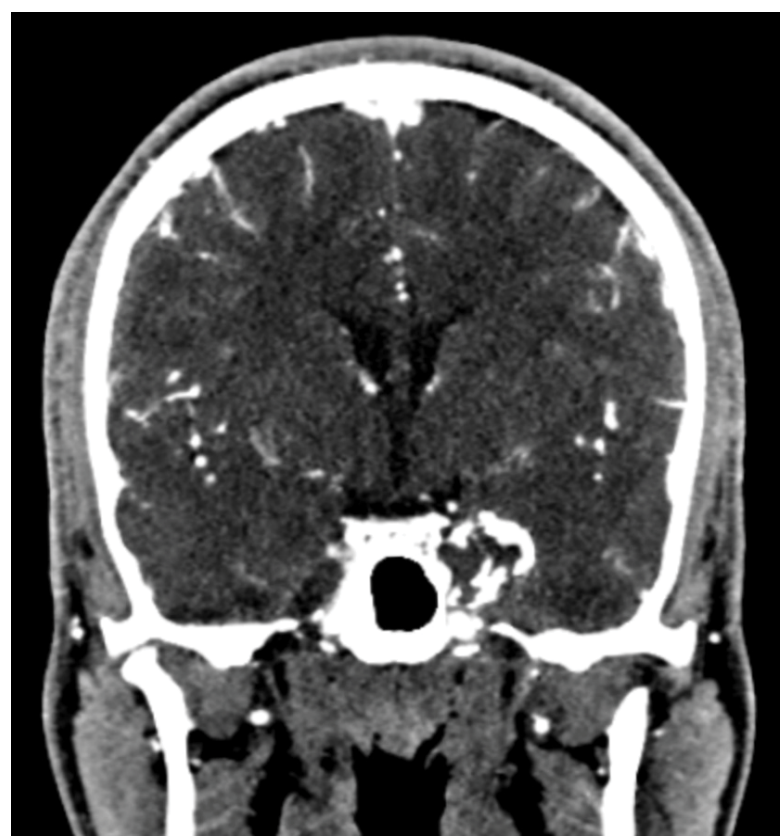

B

Figure 2: $(A, B)$ Pre-operative axial and coronal brain CT with contrast shows the partially calcified mass arising from the left cavernous sinus with mass effect on mesial temporal lobe.

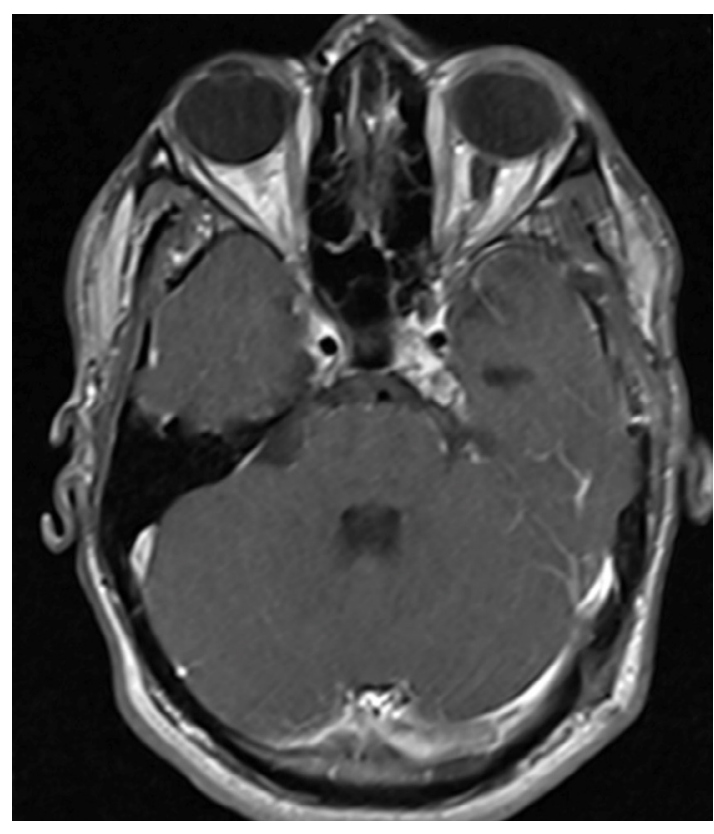

A

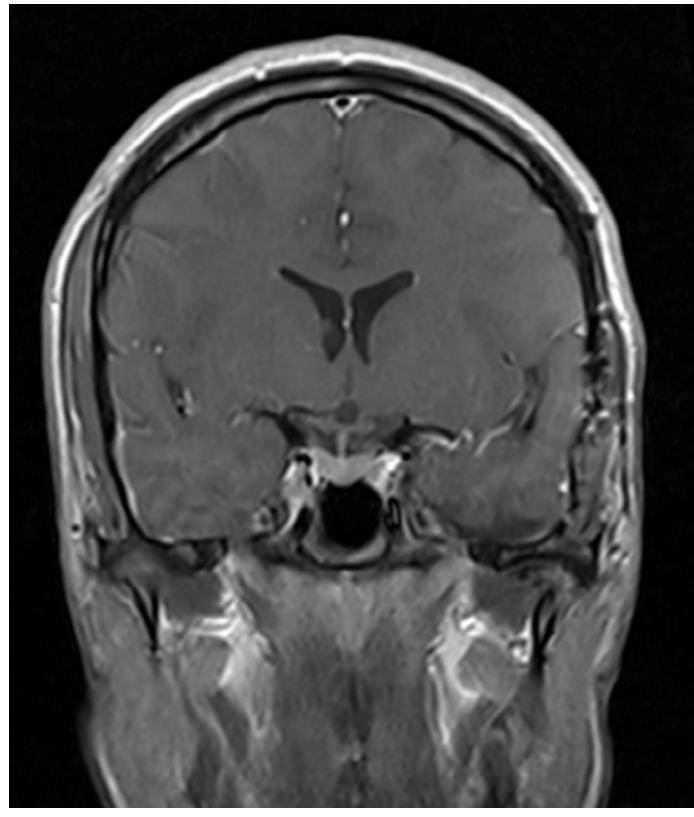

B

Figure 3: $(A, B) 12$ months post-operative axial and coronal brain MRI imaging using $T 1$ sequence with contrast shows gross total resection of the cavernous sinus mass with improved mass effect on mesial temporal lobe and ICA artery.

Patient was hospitalized and scheduled for resection of the left cavernous sinus.

\section{Treatment}

Under general anesthesia, the patient underwent resection of the left cavernous sinus mass via a combined extra-dural and intra-dural pre-temporal middle fossa skull-base approach to the cavernous sinus. Stereotactic navigation was employed to ensure accurate local- ization of the mass. A lumbar drain was placed in the cal sac with drainage of 50 ccs for relaxation of the temporal lobe prior to resection of the mass. Upon entering the tumor sac, soft grayish material as well as a heavy calcified component was noted. Internal debulking of the tumor ensued though the cavernous sinus and into the posterior fossa (Dorello's canal). Cranial nerves III, IV, V1, V2 and VI were all identified and preserved. A component of the tumor displaced the cavernous seg- 
ment of the carotid artery; the artery was carefully decompressed by further debulking of the mass. After successful successful resection of the cavernous sinus lesion, duraplaty was performed followed by cranioplasty of the pre-temporal skull defect using the autologous bone flap and titanium mesh. Throughout the procedure there were no changes in continuous SSEP, MEP and EEG neurophysiologic monitoring.

\section{Postoperative course and follow-up}

Histopathological examination of the mass revealed a benign chondroma with no signs of atypia. The patient remained hospitalized for 4 days with no complications. The lumbar drain was opened hourly to drain $10 \mathrm{ccs}$ and removed on day 3 post-surgery with total drainage of $420 \mathrm{ccs}$. An alternating eye patch for $6^{\text {th }}$ cranial nerve palsy was started post-operatively. Three-day post-operative MRI demonstrated successful resection of the cavernous sinus mass with no signs of hemorrhage or infaction.

During the three month follow up visit, there was residual diplopia and decreased sensation upper part of left forehead (V1 distribution). At the 12-month follow up visit, the patient reported persistent but much improved $\mathrm{CN}$ VI palsy with intermittent conjunctival irritation and persistent decreased sensation at left cranial nerve V1 distribution. 12-month post-op MRI noted improved decompression of the cavernous sinus and residual postoperative enhancement along left petroclinoid ligament with extension toward the cavernous sinus (Figure 3A and Figure 3B).

At the 18-month follow up visit, the patient reported resolution of the diplopia. Examination revealed near total resolution of the cranial nerve VI palsy as well as improved left cranial nerve V1 distribution sensory deficit.

\section{Conclusion}

Despite the rarity of cavernous sinus chondromas, the incidence of cranial basal chondromas is well documented; these tumors generally arise in the sellar, clival or parasellar region. Origins of basal chondromas stem from residues of primordial cartilaginous cranium replacing synchondroses. Clinical presentation of these masses varies by location; sellar chondromas may present with chiasma syndrome, quadrantanopia, decline of visual acuity and/or hypophyseal insufficiency. Parasellar and clival chondromas are often characterized by symptoms of cranial nerves III-VI disruption [14].

Cavernous sinus chondromas can be managed via application of standard cavernous sinus mass resection protocol. Initial magnetic resonance imaging should include T1-weighted and T2-weighted sequences in axial and coronal planes, fluid attenuated inversion recovery (FLAIR) sequences in the axial plane (including brain), 3D heavily T2- weighted sequences in the axial plane which aids in definition of cranial nerves, and contrast-en- hanced imaging. Additional T1-weighted fat-saturated sequences in the axial and coronal planes may aid in the discrimination of the normal cavernous sinus anatomy. Standard cavernous sinus imaging demonstrates intense homogenous enhancement following contrast administration with central flow void due to the traversing ICA, and a filling defect posterolaterally due to Gasserian ganglion in the dural recess, commonly referred to as Meckel's cave [8].

Chondromas often present as solid masses which firmly adhere to adjacent structures. Such anatomic tendencies, in addition to traversing the densely packed landscape of the cavernous sinus, pose a challenge to safe resection [15]. Major CS afferent venous channels include the sphenoparietal sinus, the superior and inferior ophthalmic veins, and the sylvian vein; while the notable efferent venous channels include the superior and inferior petrosal sinuses. The cavernous segment of the ICA and the $6^{\text {th }}$ cranial nerve traverse medially through the CS; the abducens nerve positioned inferolateral to the ICA. The oculomotor, trochlear, and ophthalmic and maxillary divisions of the trigeminal cranial nerves are positioned laterally in the cavernous sinus - anteriorly to posteriorly [16].

Due to significant improvements of the neurovascular techniques, the study of the cavernous sinus anatomy has been the object of great interest due to the wellknown advantages of the trans-cavernous approach in the treatment of selected aneurysms such as basilar tip aneurysms or posterior fossa tumors with petrosal extensions $[17,18]$.

Given its delicate anatomy, primary approaches to enter the cavernous sinus include the middle cranial fossa epidural approach, the combined transsylvian-subtemporal approach, and the medial transsphenoidal approach. The middle cranial fossa epidural approach allows for access via the Glasscock triangle and provides the greatest exposure of the lateral surface of the posterior vertical and horizontal segments of the ICA, the posterior bend, and the lower part of the anterior bend [19]. The combined transsylvian-subtemporal approach initially exposes the upper wall of the cavernous sinus and proceeds into the anterior medial CS via the triangle defined by Dolenc or to the medial CS via the triangle defined by Hakuba $[20,21]$. Access via the anteromedial and medial triangle provides exposure to the roof of the CS; while entrance via the Parkinson triangle allows access to the lateral wall of the CS [22]. The transsphenoidal approach, is nowadays almost exclusively performed endoscopically [23]. A common encounter during cavernous sinus approach involves venous bleeding requiring adequate hemostasis.

In the case presented, a cavernous sinus chondroma resection was performed in an 18-year-old male via a pretemporal trans-cavernous approach. A pre-temporal craniotomy was performed using three burr holes. 
Microsurgical resection yielded visualization of the floor of the middle cranial fossa anteriorly to the temporal pole. Extradural elevation of the temporal lobe was performed until the arcuate eminence was appreciated posteriorly.

Anterior medial extradural dissection was taken until the V1 and V2 branches of the trigeminal nerve were dissected extradurally. The calcified tumor was palpated within the petrous apex and the dura was opened in a vertical manner to enter the tumor cavity. Such an approach primarily exposes the lateral wall of the cavernous sinus and upper basilar region via removal of the anterior and/or posterior clinoid processes. Lateral expansion exposes Meckel's cave with widening possible via resection of the petrous apex to aid in further debulking of tumors within this eloquent region [17].

Cavernous sinus lesions in the pediatric population are rare; management of such tumors is not well documented in current literature. Three standard approaches to cavernous sinus mass resection include the middle cranial fossa epidural approach, the combined transsylvian-subtemporal approach, and the medial transsphenoidal approach. This case report describes successful management of cavernous sinus chondroma in a young adult via a pretemporal trans-cavernous approach and discusses standard diagnostic and management strategies for cavernous sinus resections.

\section{Declarations}

\section{Funding}

The project was completed without financial support.

\section{Conflicts of interest/Competing interests}

On behalf of all authors, the corresponding author states that there is no conflict of interest.

\section{Availability of data and material}

Data was obtained via EMR and via pubmed indexed articles for comprehensive literature review.

\section{Code availability}

N/A.

\section{Authors' contributions}

Carlos Roberto Massella Jr and Yasmeen Elsawaf wrote the manuscript and edited per the direction of Sabino Luzzi, Paulo Mácio Porto de Melo and Samer K. Elbabaa. The idea for the manuscript was led by Carlos Roberto Massella Jr, Paulo Mácio Porto de Melo and Samer K. Elbabaa and Sabino Luzzi primarily assisted with editing of the final manuscript. All authors were involved in the final approval of the manuscript.

\section{References}

1. Delgado-Lopez PD, V Martin-Velasco, AM Galacho-Harri- ero, JM Castilla-Diez, A Rodriguez-Salazar, et al. (2007) Large chondroma of the dural convexity in a patient with Noonan's syndrome: Case report and review of the literature. Neurocirugia (Astur) 18: 241-246.

2. Nakayama M, T Nagayama, H Hirano, T Oyoshi, J Kuratsu (2001) Giant chondroma arising from the dura mater of the convexity. Case report and review of the literature. J Neurosurg 94: 331-334.

3. De Coene B, C Gilliard, C Grandin, JF Nisolle, JP Trigaux, et al. (1997) Unusual location of an intracranial chondroma. AJNR Am J Neuroradiol 18: 573-575.

4. Patel A, Munthali L, Bodi I (2009) Giant cystic intracranial chondroma of the falx with review of literature. Neuropathology 29: 315-317.

5. Heo J, Cho SJ (2014) A case of giant skull base chondroma. Brain Tumor Res Treat 2: 92-95.

6. Tu YK, Tseng MY, Liu HM (2000) Experience in surgical management of tumours involving the cavernous sinus. $J$ Clin Neurosci 7: 419-424.

7. Nadarajah J, Kumble S, Ajay K, Sheragaru H, Atin K, et al. (2015) MR imaging of cavernous sinus lesions: Pictorial review. J Neuroradiol 42: 305-319.

8. Chen L, Xiang Huang, Ying Mao, Liangfu Zhou (2014) Reconsideration of cavernous sinus surgeries. Neurosurgery.

9. Duron L, Sadones F, Thiesse P, Cecile Cellier, Claire Alapetite, et al. (2018) Loco-regional extensions of central nervous system germ cell tumors: A retrospective radiological analysis of 100 patients. Neuroradiology 60: 27-34.

10. Jaimovich SG, Thea VC, Guevara M, Gardella JL (2013) Cavernous sinus tuberculoma mimicking a neoplasm: Case report, literature review, and diagnostic and treatment suggestions for tuberculomas in rare locations. Surg Neurol Int 4: 158.

11. Kumar VR, Madhugiri VS, Verma SK, Barathi SD, Yadav AK, et al. (2013) Tuberculoma of the cavernous sinus and meckel's cave in a child. Pediatr Neurosurg 49: 369-373.

12. Su J, Xianrui Yuan, Zijin Zhao, Xiangyu Wang, Junquan Wang, et al. (2016) Pretemporal transcavernous approach tailored surgery of cavernous sinus tumors: A consecutive series of 31 cases report. Zhonghua Wai Ke Za Zhi 54: 367371.

13. Terasaka S, Sawamura $Y$, Abe H (1997) Surgical removal of a cavernous sinus chondroma. Surg Neurol 48: 153-159.

14. Sepehrnia A, Samii M, Tatagiba M (1991) Management of intracavernous tumours: An 11-year experience. Acta Neurochir Suppl (Wien) 53: 122-126.

15. Aoki A, K Mori, A Tajima, M Maeda (1999) Sellar chondroma--case report. Neurol Med Chir (Tokyo) 39: 870-874.

16. Fratzoglou M, Nicolas C, Vasilios P, Dimitrios B, Melpomeni $P$ (2008) Cavernous sinus chondroma. Case report and review of the literature. Ann Ital Chir 79: 43-45.

17. Krisht AF (2005) Transcavernous approach to diseases of the anterior upper third of the posterior fossa. Neurosurg Focus.

18. Zhang R, Zhou LF, Mao Y (2005) Microsurgical treatment of nonmeningeal tumors of the cavernous sinus. Zhonghua Yi Xue Za Zhi 85: 1373-1378.

19. Dolenc VV (1989) Anatomy of the cavernous sinus. In: Anatomy and Surgery of the Cavernous Sinus. Springer, Vienna. 
20. Hakuba A, K Tanaka, T Suzuki, S Nishimurani (1989) A combined orbitozygomatic infratemporal epidural and subdural approach for lesions involving the entire cavernous sinus. J Neurosurg 71: 699-704.

21. Parkinson D (1965) A surgical approach to the cavernous portion of the carotid artery. Anatomical studies and case report. J Neurosurg 23: 474-483.

22. Zoia C, Daniele B, Gianluigi D, Sabino L, Mattia D, et al. (2019) Transnasal endoscopic removal of a retrochiasmatic cavernoma: A case report and review of literature. Surg Neurol Int 10: 76.

23. Arnaout MM, Sabino L, Renato G, Khaled A (2020) Supraorbital keyhole approach: Pure endoscopic and endoscope-assisted perspective. Clin Neurol Neurosurg 189: 105623.

24. Ceyhan M, Erdem G, Kanra G, Kaya S, Onerci M (1994) Lymphoma with bilateral cavernous sinus involvement in early childhood. Pediatr Neurol 10: 67-69.

25. Kalina P, Black K, Woldenberg R (1996) Burkitt's lymphoma of the skull base presenting as cavernous sinus syndrome in early childhood. Pediatr Radiol 26: 416-417.

26. Ganesan V, JP Lin, WK Chong, FJ Kirkham, RA Surtees (1996) Painful and painless ophthalmoplegia with cavernous sinus pseudotumour. Arch Dis Child.

27. del Toro M, Macaya A, Vazquez E, Roig M (2001) Painful ophthalmoplegia with reversible carotid stenosis in a child. Pediatr Neurol 24: 317-319.

28. Yeung M, Kwong K, Wong Y, Wong S (2004) Paediatric Tolosa-Hunt syndrome. Journal of Paediatrics and Child Health 40: 410-413.

29. Lee AG, Quick SJ, Liu GT, Rheingold SR (2003) A child- hood cavernous conundrum. Surv Ophthalmol. 49: 231236.

30. Seixas DV, Lobo AL, Farinha NJ, Cavadas L, Campos MM, et al. (2006) Burkitt leukemia with numb chin syndrome and cavernous sinus involvement. Eur J Paediatr Neurol 10: 145-147.

31. Zanus C, Furlan C, Costa P, Cosentini D, Carrozzi M (2009) The Tolosa-Hunt syndrome in children: A case report. Cephalalgia 29: 1232-1237.

32. Alioglu B, Ustun $\mathrm{H}$, Sonmez A, Kaplan H, Arikan Fl, et al. (2009) A rare presentation of central nervous system in a pediatric patient with Hodgkin disease: Cavernous sinus syndrome. J Pediatr Hematol Oncol. 31: 774-777.

33. Akinci A, Acaroglu G, Sayli TR, Demir B (2009) Cavernous sinus syndrome as the initial presentation of childhood non-hodgkin lymphoma. J Pediatr Ophthalmol Strabismus 46: $232-234$

34. Demirkaya M, Sevinir B, Ozdemir O, Nazlioglu HO, Okan M (2010) Lymphoma of the cavernous sinus mimicking Tolosa-Hunt syndrome in a child. Pediatr Neurol 42: 351-354.

35. Cerisola A, Gonzalez G, Scavone C (2011) Tolosa-Hunt syndrome preceded by facial palsy in a child. Pediatr Neurol 44: 61-64.

36. Kang NH, Lim KH, Sung SH (2011) Horner's syndrome with abducens nerve palsy. Korean J Ophthalmol. 25: 459-462.

37. Litkowski P, Khakoo Y, Gilheeney S, Souweidane M, Huse $\mathrm{J}$, et al. (2011) Hemangioma of the cavernous sinus in a child. Neurology 77: 1647-1648.

38. Benzohra DE, Damry N, Delpierre I, Huybrechts S, Monier A, et al. (2011) Tolosa-Hunt syndrome in children. JBRBTR 94: 290-292. 